\title{
Implementation and outcomes of group medical appointments in an outpatient specialty care clinic
}

Beth De Vries, MS, RN, APRN, BC, FNP (Family Nurse Practitioner) ${ }^{1}$, Cynthia Darling-Fisher, PhD, APRN, BC, FNP (Assistant Professor) $^{2}$, Anne C. Thomas, PhD, ARPN, BC, ANP (Assistant Professor) $)^{2}, \boldsymbol{\&}$

Elizabeth B. Belanger-Shugart, MS, RN, APRN, BC (Family Nurse Practitioner) ${ }^{3}$

1 Arnot Medical Services, Internal Medicine, Elmira, New York

2 University of Michigan School of Nursing, Ann Arbor, Michigan

3 Pulmonary Critical Associates, Ypsilanti, Michigan

\section{Keywords}

Group medical appointments; chronic obstructive pulmonary disease; nurse practitioner.

\section{Correspondence}

Beth De Vries, MS, RN, APRN, BC, FNP, 602 Ivy Street, Elmira, NY 14905.

Tel: 607-735-4633; Fax: 607-735-4628;

E-mail: bdevries@aomc.org

Received: April 2007;

accepted: July 2007

doi:10.1111/j.1745-7599.2007.00300.x

\begin{abstract}
Purpose: To describe the outcomes of a nurse practitioner (NP)-facilitated group medical appointment (GMA) intervention for chronic obstructive pulmonary disease (COPD) clients in a pulmonary practice in the Midwest.

Data sources: Medical records from a convenience sample of six established pulmonary patients in a Midwest specialty clinic who received care in a group format were retrospectively audited. Outcome measures included examination of changes in the utilization of healthcare services, exercise tolerance, and use of nonpharmacological and pharmacological interventions pre- and postparticipation in the GMA program.

Conclusions: NP-facilitated GMAs are feasible and can help improve health outcomes. Results showed a significant increase in exercise tolerance measured by 6 -min walk distance. Anecdotally, patient and provider response to the GMA was very positive.

Implications for practice: The GMA format is an innovative solution for the management of chronic disease patients that is comprehensive, time efficient, reimbursable, and well suited to NP practice. GMAs can be implemented in most practice settings.
\end{abstract}

\section{Introduction and significance}

Chronic illness is a major public health concern contributing to death, illness, and disability of many Americans. More than 90 million Americans are living with one or more chronic illnesses, and these chronic illnesses cause $70 \%$ of all deaths in the United States and account for more than $75 \%$ of the nation's medical care costs (Centers for Disease Control and Prevention [CDC], 2005). Chronic conditions cause major limitations in activity for more than 10\% of Americans. Chronic obstructive pulmonary disease (COPD) was the fourth most common cause of death in 1999, and respiratory problems were the fourth leading cause of disability among persons aged 15 years or older in the United States during 1991 and 1992 (CDC). In 2000, 119,000 deaths, 726,000 hospitalizations, and 1.5 million hospital emergency department visits were caused by COPD (CDC). The prevalence, morbidity, and mortality from COPD are expected to rise especially in countries with rapidly aging populations (Feenstra, van Genugten, Hoogenveen, Wouters, \& Rutten-van Molken, 2001).

Patients with chronic diseases, like COPD, require intense patient education, counseling, lifestyle modification, and complicated pharmacological management, all of which take a significant amount of provider time. These interventions are difficult to achieve in the current healthcare system where less time per patient visit is a result of increasing numbers of patients seen per day. Historically, medical institutions have been structured to treat acute episodic health problems; however, they are poorly equipped to handle chronically ill patients who require complex services (Wellington, 2001).

The group medical appointment (GMA) offers an alternative structure to deliver more effective and efficient care 
to chronically ill patients (Schmucker, 2006). GMAs provide patients with increased interaction time with their provider. A group of 8-12 patients is able to spend $90 \mathrm{~min}$ interacting with their provider instead of the usual 10- to 15-min individual appointment. GMAs incorporate all the components of individual appointments, including oneon-one medical evaluations, but allow more time for patient education and promoting self-management (Jaber, Braksmajer, \& Trilling, 2006).

GMAs have been widely used and studied with patients with diabetes in primary care settings. GMAs have improved adherence to standards of care, promoted a sense of trust in the provider (Clancy, Brown, Magruder, \& Huang, 2003), demonstrated significant decreases in glycosylated hemoglobin (HbAlc) levels, increases in selfefficacy, increases in satisfaction with care, and lowered frequency of hospitalizations (Sadur et al., 1999) and show positive outcomes with culturally and economically diverse populations (Culhane-Pera et al., 2004; Wagner et al., 2001). Trento et al.'s (2004) 5-year randomized control trial documented significant increases in patient problem-solving ability, stable HbAlc levels, increased quality of life, increased diabetes knowledge, and decreased use of hypoglycemic agents.

Patients with chronic diseases other than diabetes have also benefited from GMAs. Older adults with various chronic diseases treated in primary care GMAs had decreased inpatient admissions, decreased emergency room visits, and increased quality of life, self-efficacy, and satisfaction with care (Coleman et al., 2001; Scott et al., 2004). In women's health care, GMAs have resulted in increased provider productivity and decreased urgent care visits (Miller, Zantop, Hammer, Faust, \& Grumbach, 2004; Thacker, Maxwell, Saporito, \& Bronson, 2005). Benefits of GMAs have also been documented in specialty populations such as coronary artery disease clients (Masley, Phillips, \& Copelan, 2001), headache sufferers (Blumenfeld \& Tischio, 2003; Maizels, Saenz, \& Wirjo, 2003), and urological patients (Fletcher, Clark, Overstreet, \& Steers, 2006).

There have been few studies showing the use of the GMA format with COPD patients. Gallefoss and Bakke (2000) implemented nurse-led group education sessions with a focus on self-management with COPD and asthma patients. Group education sessions (separate from the medical visit) were used to improve outcomes in COPD patients. The intervention group had 2-h educational sessions rather than the GMA format. The patients otherwise received the usual medical care. The intervention significantly reduced office visits and improved quality of life. Another study showed that monthly nurse-led educational sessions and medical consultation with COPD patients in a specialty practice significantly reduced hospital admissions and length of hospital stay (Soler et al., 2006).

The studies described above indicate that patients with chronic illnesses have greatly benefited from implementation of GMAs in various clinical settings. Although there have been studies using group educational interventions in COPD patients, none have used the GMA format. Patients with COPD require complicated medical care comparable to that of diabetes patients; therefore, GMAs could potentially improve outcomes in COPD. In addition, COPD patients are a unique population presenting different needs and challenges for healthcare providers. To design an effective approach for these patients, it is helpful to consider Yalom's group model and curative factors.

\section{Theoretical framework}

The studies described above demonstrate the feasibility and positive outcomes of GMAs, but few hypothesize why GMAs work so well. Yalom's (1995) description of group psychotherapy provides a basis for understanding the benefits of group work and for designing group interventions. Yalom describes 11 curative factors of group therapy. Curative factors are interpersonal experiences that promote a sense of well-being, productivity, and competence. Curative factors have also been described in groups of older adults (Burnside $\&$ Ranney, 2005). Four factors, instillation of hope, universality, altruism, and group cohesiveness, are particularly applicable to GMAs involving older adults with chronic disease.

Group visits may help instill hope by offering increased education, promoting effective coping skills, and receiving encouragement from other group members. Participants in disease-specific GMAs appreciate the universality of their experiences with that chronic disease through sharing. GMAs also offer patients an opportunity to feel useful, that is, altruistic. Group discussions provide older adults the opportunity to share their knowledge, which, in turn, gives individuals a sense of pride by being able to help others. Group cohesiveness is the togetherness and camaraderie the group develops over time through problem solving, which gives individual members a feeling of belonging to the group (Schmucker, 2006).

Yalom's curative factors were useful in explaining the benefits of group care seen in this study. When medical care is provided in a group format, the group itself is part of the healing. These principles are especially applicable to the COPD patients in this study. Because of their disease and the impact it may have on their lives, these older adults may feel particularly hopeless, isolated, worthless, and alone. Yalom's curative factors offer a framework for 
understanding the underlying benefits of GMAs with COPD patients.

\section{Methods}

\section{Design}

This pilot study used a retrospective de-identified chart audit to collect demographic data and outcome measures for participants in the GMA and was considered exempt by institutional review. Data were collected on the patient's health outcomes before and after participating in GMAs for 6 months. Outcome measures included examination of changes in the utilization of healthcare services, exercise tolerance, and use of nonpharmacological and pharmacological interventions pre- and postparticipation in the GMA program. Chart data collected included demographics, medical diagnosis, assessments, and data for usual standard of care including emergency room (ER) visits, hospital admission, office visits, participation in pulmonary rehabilitation, smoking status, depression screening, and medication use.

Data were analyzed with SPSS 13.0 to determine changes in the outcomes pre- and post-GMA participation, as well as demographic data including distribution of age, race, sex, marital status, education level, time since diagnosis, oxygen use, and disease classification according to the Global Initiative for Chronic Obstructive Lung Disease (GOLD) standards (Pauwels et al., 2001).

\section{Sample}

Study participants were drawn from a convenience sample of established pulmonary patients in a specialty clinic in the Midwest who expressed interest in receiving care in a group format. The GMA format was introduced at an educational session about living well with COPD. Patients interested in the GMA wrote their names and addresses on a card and handed it to the speaker. These patients were sent an informational package in the mail and informed of the first GMA date and time. Of the 10 patients who expressed interest, 6 were able to attend the first GMA and had complete data.

\section{Procedure}

The GMAs were held monthly for the first 3 months and then planned for every 3 months for the next year. They were scheduled for 90-min sessions with 15 min allowed for introductions, 30-45 min devoted to discussion and education, and $15 \mathrm{~min}$ allotted for discussion of selfmanagement action plans. Participants were individually seen by the nurse practitioner (NP) in a quiet location in the room during the group session for a short time period.
The patients were sent packets in the mail before the first visit. Packets included an introduction letter, a question and answer sheet about group visits, and a planned care visit form. The planned care form included a review of systems, past medical history, family history, social history, current medications, and depression screening with the two-question Patient Health Screening Questionnaire (PHQ)-2. The patients were to fill out the planned care form before the scheduled visit and bring it in with them. Positive screening on the PHQ-2 was followed up with PHQ-9 screening during the visit. Patients wrote updates on the planned care visit form for subsequent visits.

During the first visit, confidentiality, group visit norms, self-management, and action plans were reviewed. The patients were asked during the first group what topics they would like to discuss and the NP chose topics from their lists. Educational topics discussed included living with COPD, review of the disease process, medications, and the importance of physical activity. Patients were given educational handouts for every topic and a binder to organize the information. Patients also received pedometers, graphs to track their step counts, and an exercise Theraband ${ }^{\circledR}$ during the physical activity session.

The staff participating in the GMA included the NP, a registered nurse $(\mathrm{RN})$, two respiratory therapists (RT), a student NP, and two medical assistants. All the staff members did not participate in every GMA. The NP and an RN or a medical assistant facilitated all the GMAs. The RT staff helped gather 6-min walk (6MW) data and provided some of the education. The $\mathrm{RN}$ and student NP helped facilitate discussions, while the NP was seeing patients on an individual basis. The medical assistants helped with vital signs, paperwork, and discussions.

\section{Results}

\section{Demographics}

Participants in the study $(n=6)$ were established patients with COPD at a local pulmonary specialty practice. The majority of the patients in the study were older Caucasian adults with severe COPD. Most of the patients had been diagnosed with COPD more than 5 years ago, but one participant was diagnosed within the last year. Mean time since diagnosis was 6.5 years $(S D=3.8)$. Four participants were male, all were Caucasian, five were married, one widowed, and all were ex-smokers. The mean age of the participants was $67(\mathrm{SD}=9.23)$. Five participants had severe COPD and one had moderate COPD according to the GOLD standard classification. Attendance at the four offered GMAs was tallied: four of the participants attended $100 \%$ of the offered GMAs, 
one attended $75 \%$ of the appointments, and one attended $50 \%$ of the appointments. The two participants who missed appointments were hospitalized when the GMA visit was scheduled.

\section{Utilization of healthcare services}

There were no significant differences in utilization of healthcare services found in this study. Utilization was measured 3 months before and 3 months after the GMAs. The number of visits to the ER was unchanged. Number of hospital admissions was equal except for one participant who had no admissions 3 months before and three admissions in the 3 months post-GMA. There were two participants who were hospitalized during the GMA study and one was in the hospital for more than 4 weeks. The number of office visits among participants increased and in some participants doubled. GMA participants had monthly appointments compared to the standard individual appointment every 3-6 months.

\section{Nonpharmacological and pharmacological measurements}

Participation in pulmonary rehabilitation, smoking status, depression screening, inhaled bronchodilator use, and oral corticosteroid use was measured 3 months before and 3 months after the GMAs. Results are outlined in Table 1. An important result was the increase in depression screening that was included on the planned visit form. Two of the participants had not been screened recently and had a positive PHQ-2, which led to further screening and referrals. There was no change in inhaled bronchodilator use. Oral corticosteroid use increased in one participant following a lengthy hospitalization.

\section{Significant improvement in exercise tolerance}

The 6MW distance was measured on all participants at the first GMA and then 5 months later after four GMAs. There was an average of $62 \%$ improvement in $6 \mathrm{MW}$ distance in the group. All the participants had some improvement; the smallest improvement was $29 \%$ and the biggest improvement was 103\%. See Table 2 for complete data. There was a significant difference between the

Table 1 Nonpharmacological and pharmacological measurements

\begin{tabular}{lcc}
\hline & Pre-GMA (\%) & Post-GMA (\%) \\
\hline Participation in pulmonary rehabilitation & 50 & 50 \\
Smoking status addressed & 100 & 100 \\
Depression screening & 50 & 100 \\
Scheduled inhaled bronchodilator & 67 & 67 \\
Oral corticosteroid use $>2 \times /$ year & 33 & 50 \\
\hline
\end{tabular}

Table 2 6MW data

\begin{tabular}{llll}
\hline & \multicolumn{2}{l}{ 6MW (feet) } & \\
\cline { 2 - 3 } Participant & Pre-GMA & Post-GMA & Improvement (\%) \\
\hline 1 & 982 & 1530 & 56 \\
2 & 650 & 1203 & 85 \\
3 & 774 & 1323 & 71 \\
4 & 660 & 1340 & 103 \\
5 & 580 & 750 & 29 \\
6 & 825 & 1080 & 31 \\
\hline
\end{tabular}

pre-GMA 6MW distance $(M=745.2$ feet, $\mathrm{SD}=146.2)$ and the post-GMA $6 \mathrm{MW}$ distance $(\mathrm{M}=1204.3 \mathrm{feet}, \mathrm{SD}=$ $268.45)$ with the post-GMA 6MW distance being farther, $t(6)=2.57, p<0.001$.

\section{Discussion}

Results of this outcomes study demonstrate how the treatment of COPD can be easily incorporated into GMA format of care with positive outcomes. The NP was able to complete physical exams, order lab and diagnostic testing, and adjust medications as needed. There was time for education, a pivotal part of providing care for COPD patients, and the GMA format is ideal for increasing education time.

Positive trends in patient outcomes after implementation of the GMA format were noted in this study. Despite the small sample size and a short time frame, there was significant improvement in functional abilities of the participants as demonstrated by improved 6MW distances. Improved exercise tolerance helps to improve symptoms and quality of life for COPD patients. The improvement was evident in patients with severe disease and even in the patients who were not able to attend all the GMAs.

The improvement in 6MW distance is an exciting result; however, several of the outcomes variables did not improve. Many of these may be related to the severity of the patients' illness. The patients did not have fewer visits to the ER or fewer hospital admissions. They were seen more often in the office setting with the GMAs. Therefore, the cost of care with more frequent office visits was increased. The use of medications to control COPD was unchanged in most of the sample and increased in one patient. Most of the participants in the study had severe COPD and were expected to require a high level of medical care. The participants in the study were patients who expressed interest in the GMA as a modality to learn more about their disease. Patients with more severe disease may have been more concerned about their disease and more motivated to join the GMA. 
There were many factors that may have influenced the improved outcomes. The GMA format provided increased time for education and allowed the NP to focus on the importance of exercise and how COPD patients can increase their exercise. The group also had education from the RT who also taught breathing exercises. Patients benefited from receiving pedometers and Therabands ${ }^{\circledR}$ to help incorporate exercise into their lives. As part of the GMA, patients committed to action plans, which were short-term goals they wanted to achieve. Many of them chose goals to increase physical activity. There is also an intrinsic value of the group format, which has been described using Yalom's curative factors.

Yalom's curative factors were observed in the group behavior. The group members gained universality realizing that the other members were coping with similar challenges. Anecdotally, the NP coordinating the GMAs observed participants supporting their peers in the group to make lifestyle changes and increase activity. Some of the participants set high goals for physical activity and this encouraged other members. They also had the opportunity to share their knowledge and had a feeling of usefulness or altruism. During one of the GMAs, a patient asked about traveling with oxygen and another patient explained how easy it was to travel. The NP may have been able to provide the same information but not the firsthand experience and the reassurance that it would not be complicated. The participants bonded with each other and had a sense of belonging and cohesiveness in the group. The patients could see how their peers were successfully coping with COPD and finding ways to live and enjoy their life.

Learning to cope with COPD was one important psychosocial concern of the group. Depression was another significant concern, as it often accompanies chronic disease, specifically COPD, and may go undiagnosed because of other pressing medical concerns (Cicutto, Brooks, $\&$ Henderson, 2004). Use of the depression screening instrument on the planned care form helped to identify two patients in the group who were depressed and had not been receiving treatment. Information gained from the depression screening was sent back to the primary care clinician, and the patients were referred for further treatment that led to identification and treatment of depression and substance abuse.

Patient satisfaction was not surveyed in this study, but other studies have shown increased patient satisfaction with GMAs. However, group behavior in this study suggested that these patients felt positively about their group experience. When the NP tried to transition the group to every 3 months instead of monthly groups, the patients resisted the change and requested monthly meetings.
The patients looked forward to the next meeting and what they would learn.

\section{Limitations}

This project had limitations inherent in any small, nonrandomized clinical study. In addition, the participants were self-selected and motivated to learn more about their disease and how to manage it. The patients' motivation may have skewed the results. Also, the sample included only Caucasian participants and this limits its generalizability to other populations.

\section{Implications for future research}

This study supports the feasibility of and potential benefit for using GMAs with pulmonary patients in a specialty practice. However, larger randomized controlled trials over longer periods of time with pulmonary patients are needed to draw more conclusions about the direct benefits of GMAs in pulmonary specialty practice. Qualitative research and focus groups could offer insight from patients about specific aspects of group care and the patients' perspective on the experience. Quantitative and qualitative research with patient satisfaction, quality of life, functional status measurements, self-efficacy and ability to self-manage chronic disease may offer additional insights into quality of care with GMAs. Use of Yalom's curative factors as a theoretical framework in future research would help to explain the significant benefit of providing care in the group setting. This knowledge can also help in the planning of group sessions and activities.

\section{Recommendations for practice}

Group appointments can work in many different situations but must be tailored to the individual practice needs. It is very important to have the practice administration and other members of the team involved in and supporting the GMAs. Starting group visits requires planning and additional time. The office staff who answer the phones and greet the patients also need to be educated about how to explain GMAs. Advertising in the office and recruiting patients are important to obtain appropriate group size and maintenance of the group (Schmucker, 2006). At least six patients are required for a 90-min group to be cost effective as the NP in this practice would normally see four to six patients individually in $90 \mathrm{~min}$.

Patients in the group valued the extra time with the provider and this needs to be considered in the functioning of the group. The group format adapted for this study pulled patients out of the group for individual consultation. While the NP provider was engaged with an 
individual, discussion and education were led by other members of the team. In this system, there were questions that the discussion leaders could not answer, and the patients lost some interaction time with the NP. This was not ideal from the participants' perspective. The NP also felt she missed some important information about the patients during the time she was out of the group. Also, some concerns discussed in the participant's individual time were relevant for the entire group. Because of these factors, the NP changed the format of the GMA for the next group of participants. The new group format used was organized to have patients' concerns addressed within the group, physical exams performed around the table, and individual time offered only as needed. The patients were open to sharing in the group and had very similar medical problems, which allowed for more education. The new group format also decreased the number of staff required to facilitate the group.

Documentation of the visit should be completed during the GMA to utilize the provider's time effectively. Developing a documentation form that includes a check box or circle format allows the provider to quickly document the important points of the exam, while the facilitator leads the discussion. It is important to have a good facilitator who can help maintain the flow of the group, while the provider completes these tasks. At the end of the 90-min visit, the provider should have very little paperwork to complete. This is important because the GMA requires some preparation time that would not be cost effective if the provider had to complete documentation after the visit. Lab requisitions and prescription refills can also be completed during the GMA.

\section{Conclusion}

Chronic disease affects many Americans today and is a growing problem that is overwhelming the current medical care system. These illnesses require emotional and social adaptation, lifestyle change, and self-management for optimal outcomes. The GMA format is an innovative solution for the management of chronic disease patients that is well suited to NP practice, comprehensive, time efficient, and reimbursable. Many NPs are experienced with providing education and counseling and value that part of their practice. This format allows NPs to use their strengths as educators more efficiently because they can provide quality education to several patients in less time. Providing high-quality care, seeing improved outcomes, and providing care in a more relaxed environment may increase provider satisfaction. This pilot study showed that an NP-facilitated GMA intervention with COPD patients in specialty pulmonary practice was feasible for providers and beneficial to patients.

\section{Acknowledgments}

The authors would like to thank the Pulmonary Critical Care Associates staff at Saint Joseph Mercy Hospital and Kay Dwyer, RN, at Huron Valley Physicians Association for their assistance with this study.

\section{References}

Blumenfeld, A., \& Tischio, M. (2003). Center of excellence for headache care: Group model at Kaiser Permanente. Headache, 43, 431-440.

Burnside, I., \& Ranney, M. (2005). Principles of Yalom. In B. Haight \& F. Gibson (Eds.), Burnside's working with older adults: Group process and techniques (4th ed., pp. 47-55). Boston: Jones and Bartlett.

Center for Disease Control and Prevention. (2005). National Center for Chronic Disease Prevention and Health Promotion. Chronic disease overview. Retrieved November 13, 2006, from http://www.cdc.gov/nccdphp/overview.htm

Cicutto, L., Brooks, D., \& Henderson, K. (2004). Self-care issues from the perspective of individuals with chronic obstructive pulmonary disease. Patient Education and Counseling, 55, 168-176.

Clancy, D., Brown, S., Magruder, K., \& Huang, P. (2003). Group visits in medically and economically disadvantaged patients with type 2 diabetes and their relationships to clinical outcomes. Topics in Health Information Management, 24(1), 8-14.

Coleman, E., Eilertsen, T., Kramer, A., Magid, D., Beck, A., \& Conner, D. (2001). Reducing emergency visits in older adults with chronic illness: A randomized, controlled trial of group visits. Effective Clinical Practice, 4(2), 49-57.

Culhane-Pera, K., Peterson, K., Crain, A. L., Center, B., Maysen, L., \& Her, B., et al. (2004). Group visits for Hmong adults with type 2 diabetes mellitus: A pre-post analysis. Journal for Health Care for the Poor and Underserved, 16, 315-327.

Feenstra, T. L., van Genugten, M. L., Hoogenveen, R. T., Wouters, E. F., $\delta$ Rutten-van Molken, M. P. (2001). The impact of aging and smoking on the future burden of chronic obstructive pulmonary disease: A model analysis in the Netherlands. American Journal of Respiratory Critical Care Medicine, 164, 590-596.

Fletcher, S., Clark, S., Overstreet, D., \& Steers, W. (2006). An improved approach to follow-up care for the urological patient: Drop-in group medical appointments. Journal of Urology, 176, 1122-1126.

Gallefoss, F., \& Bakke, P. (2000). Impact of patient education and self-management on morbidity in asthmatics and patients with chronic obstructive pulmonary disease. Respiratory Medicine, 94, 279-287.

Jaber, R., Braksmajer, A., \& Trilling, J. (2006). Group visits: Models, benefits, and challenges. Family Practice Management, 13(1), 37-40.

Maizels, M., Saenz, V., \& Wirjo, J. (2003). Impact of a group-based model of disease management for headache. Headache, 43, 621-627.

Masley, S., Phillips, S., \& Copelan, J. (2001). Group office visits change dietary habits of patients with coronary artery disease: The dietary intervention and evaluation trial (D.I.E.T.). Journal of Family Practice, 50, 235-239.

Miller, D., Zantop, V., Hammer, H., Faust, S., \& Grumbach, K. (2004). Group medical visits for low-income women with chronic disease: A feasibility study. Journal of Women's Health, 13, 217-225.

Pauwels, R. A., Buist, A. S., Ma, P., Jenkins, C. R., Hurd, S. S.; GOLD Scientific Committee. (2001). Global strategy for the diagnosis, management and prevention of chronic obstructive pulmonary disease. NHLBI/WHO Global Initiative for Chronic Obstructive Lung Disease (GOLD) Workshop Summary. American Journal of Respiratory Critical Care Medicine, 163, 1256-1276.

Sadur, C., Moline, N., Costa, M., Michalik, D., Mendlowitz, D., \& Roller, S., et al. (1999). Diabetes management in a Health Maintenance Organization: Efficacy of care management using cluster visits. Diabetes Care, 22, 2011-2017.

Schmucker, D. (2006). Group medical appointments: An introduction for health professionals. Sudbury, MA: Jones and Bartlett.

Scott, J. C., Conner, D. A., Venohr, I., Gade, G., McKenzie, M., \& Kramer, A. M., et al. (2004). Effectiveness of a group outpatient visit model for chronically ill older HMO members: A 2-year randomized trial of the cooperative health care clinic. Journal of the American Geriatric Society, 52, 1463-1470. 
Soler, J., Martinez-Garcia, M., Roman, P., Orero, R., Terrazas, S., \& MartinezPechuan, A. (2006). Effectiveness of a specific program for patients with chronic obstructive pulmonary disease and frequent exacerbations. Archivos de Bronconeumologia, 42, 501-508.

Thacker, H., Maxwell, R., Saporito, J., \& Bronson, D. (2005). Shared medical appointments: Facilitating interdisciplinary care for midlife women. Journal of Women's Health, 14, 867-870.

Trento, M., Passera, P., Borgo, E., Tomalino, M., Bajardi, M., \& Cavallo, F., et al. (2004). A 5-year randomized controlled study of learning, problem solving ability and quality of life modifications in people with type 2 diabetes managed by group care. Diabetes Care, 27, 670-675.

Wagner, E., Grothaous, L., Sandhu, N., Galvin, M., McGregor, M., \& Artz, K., et al. (2001). Chronic care clinics for diabetes in primary care: A system-wide randomized trial. Diabetes Care, 25, 695-700.
Wellington, M. (2001). Stanford health partners: Rationale and early experiences in establishing physician group visits and chronic disease self-management workshops. Journal of Ambulatory Care Management, 24(3), 10-16.

Yalom, I. (1995). The theory and practice of group psychotherapy (4th ed.). New York: Basic Books.

\section{Conflict of interest disclosure}

No relationship exists between any of the authors and any commercial entity or product mentioned in this article that might represent a conflict of interest. No inducements have been made to submit this article. 